\section{Antiemetic Guidelines: Not Just for the HEC of It}

Kelsey A. Klute, MD

$\mathbf{N}^{2}$ ausea and vomiting are among the most feared complications of chemotherapy. ${ }^{1}$ Clinicians often reassure people starting chemotherapy that, "It's not like on TV. We have better medicines to manage nausea and vomiting now. We don't see many patients suffering with nausea and vomiting after chemotherapy these days."

The first part of that assurance is true. We now have a variety of highly effective medications to prevent and manage nausea and vomiting today that we did not have a decade ago. Advances in the approach to prevention and management of chemotherapy-induced nausea and vomiting (CINV) allow patients to receive chemotherapy in the outpatient setting and minimize interruption of their daily lives. The impact of this advancement was recognized by both patients and physicians who voted the FDA's approval of ondansetron in 1991 and other antiemetics in subsequent years as one of the "Top 5 Advances in 50 Years of Modern Oncology" in ASCO's 50th anniversary poll in $2014 .^{2}$

The second half of the assurance, however, is misleading. We say it because we vastly underestimate the incidence of nausea in patients receiving chemotherapy. ${ }^{3}$ Approximately $50 \%$ of patients receiving highly emetogenic chemotherapy (HEC) experience some degree of nausea or vomiting, ${ }^{3-5}$ and uncontrolled CINV has been associated with reduced quality of life, increased healthcare utilization, and missed work, with a significant impact on daily functioning. ${ }^{4}$

Clinical practice guidelines published by $\mathrm{NCCN}^{6}$ and $\mathrm{ASCO}^{7}$ have established standard approaches to the prevention of CINV. Each set of guidelines outlines antiemetic treatment according to the emetogenic potential of a given regimen. HEC, defined as chemotherapy that causes acute emesis in $>90 \%$ of patients in the absence of adequate prophylaxis, includes regimens containing anthracycline and cyclophosphamide (AC), cisplatin, or carboplatin at an area under the curve of $\geq 4$. Although certain elements of both guidelines are based on expert consensus, the prophylaxis guidelines for patients receiving HEC are largely evidence-based ${ }^{5,8}$ and generally include a 3-drug regimen containing a neurokinin-1 receptor antagonist (NK1 RA), a serotonin type-3 receptor antagonist, and dexamethasone with or without olanzapine.

In this issue of JNCCN, Roeland et al ${ }^{9}$ used a large population database to query medical oncologists' rate of compliance with antiemetic prophylaxis guidelines for HEC. They found that only $35 \%$ and $58 \%$ of clinicians were $>90 \%$ adherent to antiemetic guidelines when prescribing a patient's first cycle of cisplatin or AC. They observed significant variability in guidelines adherence among individual physicians and found that the most common reason for nonadherence was omission of an NK1 RA. Though understanding the reasons for deviating from a guideline-based approach to antiemesis is beyond the scope of their study, various factors were likely at play, including underestimation of the incidence of delayed chemotherapyinduced emesis, ${ }^{3}$ lack of guideline awareness, toxicity concerns, and lack of reimbursement for antiemetic drugs.

Physicians in this study possibly intentionally selected a nonadherent prophylactic regimen based on individual patient risk factors, which have been described in the literature. It has been well-established that female sex, age $<55$ years, history of motion or morning sickness, and history of no alcohol use can increase an individual's

\section{See page 676 for related article.}

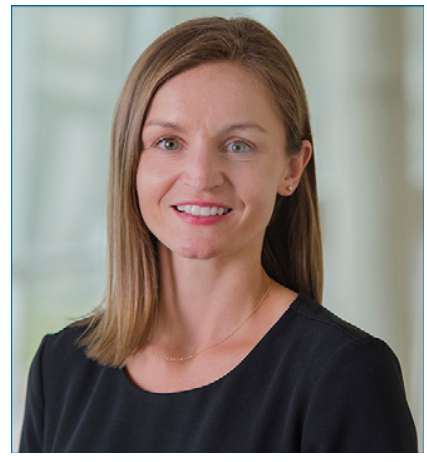

KELSEY A. KLUTE, MD

Kelsey A. Klute, MD, is a medical oncologist and Assistant Professor of Medicine at the University of Nebraska Medical Center in Omaha, Nebraska. She holds an appointment in the Gastrointestinal Cancer Program at the Fred \& Pamela Buffett Cancer Center where she is involved in both clinical and translational research.

doi: $10.6004 /$ jnccn.2020.7589

The ideas and viewpoints expressed in this commentary are those of the author and do not necessarily represent any policy, position, or program of NCCN. 
risk of CINV. ${ }^{10}$ In a clinical trial that evaluated a risk-modelguided approach to adding aprepitant with or without olanzapine to dexamethasone and a serotonin type-3 receptor antagonist in patients receiving $A C$ for early-stage breast cancer, the risk-model-guided approach was more effective than physician antiemetic choice in controlling acute and delayed CINV. ${ }^{11}$ The rate of NK1 RA or olanzapine use in the control group, however, was exceptionally low. Therefore, although the risk-model-guided approach was more effective than physician choice, it was compared with suboptimal antiemetic prophylaxis and should not be considered a standard approach.

The goal for management of CINV should be complete control of both vomiting and nausea, but it's clear that we consistently underestimate the incidence of nausea and vomiting-particularly delayed nausea-in our patients. ${ }^{3}$ Randomized trials have primarily focused on control of vomiting or complete response to antiemetic prophylaxis, defined as no vomiting and no use of rescue medications. ${ }^{12}$ Although the objective nature of complete response is appealing as a scientific endpoint, it does not adequately capture the incidence of nausea, which is more common than vomiting and has a more significant impact on quality of life. ${ }^{4}$ In one survey, although $71 \%$ of patients experienced nausea after administration of chemotherapy, only $57 \%$ of those patients used any rescue medication. ${ }^{13}$ These results highlight the problematic nature of using "complete response" as a clinical trial endpoint. "Complete control," defined as "no vomiting, no significant nausea, and no use of rescue medications," is often used as a secondary outcome measure, but it may better characterize the patient experience.

Roeland et al's 9 work did not examine the impact that nonadherence with antiemetic guidelines had on patient experiences with CINV. The data from randomized trials that informed antiemetic prophylaxis guidelines for HEC suggest that even the best approach can fall short for a proportion of patients. For example, the addition of aprepitant to ondansetron and dexamethasone in patients receiving cisplatin $\geq 70 \mathrm{mg} / \mathrm{m}^{2}$ improved the rate of complete response from $52.3 \%$ to $72.7 \%(P<.001) .{ }^{8}$ Although this is a clinically and statistically significant improvement in antiemesis management, it still left nearly $30 \%$ of patients with episodes of vomiting or nausea that required rescue medication. Thus Roeland et al's ${ }^{9}$ finding that omission of an NK1 RA accounted for most nonadherent cycles likely impacted the rate of CINV.

Further research is needed to define the rationale for and barriers to adherence with antiemetic prophylaxis guidelines to optimize both systems-based and personalized approaches to improving antiemetic prophylaxis. Patient-centered endpoints in antiemetic trials and the use of patient-reported outcomes to assess efficacy of antiemetic therapies and effect on quality of life will be essential to improve on the current approach to CINV in the future.

Despite significant advances in antiemetic prophylaxis, Roeland et $\mathrm{al}^{9}$ show that adherence to guidelines for antiemetic prophylaxis among oncologists prescribing $\mathrm{HEC}$ is low. CINV remains a significant issue for patients that is consistently underestimated by clinicians. ${ }^{3}$ Outside the context of a clinical trial, selection of antiemetic prophylaxis based on a regimen's risk of emesis remains the standard of care.

So, it's true when we say, "It's not like you see on TV. We have better medicines to manage nausea and vomiting these days." But even our most effective antiemetics can only help the patients whose doctors prescribe them.

Disclosures: The author has disclosed that she has not received any financial consideration from any person or organization to support the preparation of this article.

Correspondence: Kelsey A. Klute, MD, 987680 Nebraska Medical Center, Omaha, NE 68198-7680. Email: Kelsey.klute@unmc.edu

\section{References}

1. Coates A, Abraham S, Kaye SB, et al. On the receiving end-patient perception of the side-effects of cancer chemotherapy. Eur J Cancer Clin Oncol 1983;19:203-208.

2. ASCO. ASCO 50th Anniversary Poll Names the Top 5 Advances From the Past 50 Years. Accessed April 24, 2020. Available at: https://www. asco.org/about-asco/press-center/news-releases/asco-50th-anniversarypoll-names-top-5-advances-past-50-years

3. Grunberg SM, Deuson RR, Mavros P, et al. Incidence of chemotherapyinduced nausea and emesis after modern antiemetics. Cancer 2004;100: 2261-2268.

4. Navari RM, Aapro M. Antiemetic prophylaxis for chemotherapy-induced nausea and vomiting. N Engl J Med 2016;374:1356-1367.

5. Navari RM, Qin R, Ruddy KJ, et al. Olanzapine for the prevention of chemotherapy-induced nausea and vomiting. N Engl J Med 2016;375: 134-142.

6. Ettinger DS, Berger MJ, Barbour S, et al. NCCN Clinical Practice Guidelines in Onocology: Antiemesis. Version 2.2020. Accessed May 8, 2020. To view the most recent version, visit NCCN.org.

7. Hesketh PJ, Kris MG, Basch E, et al. Antiemetics: American Society of Clinical Oncology clinical practice guideline update. J Clin Oncol 2017; 35:3240-3261.

8. Hesketh PJ, Grunberg SM, Gralla RJ, et al. The oral neurokinin-1 antagonist aprepitant for the prevention of chemotherapy-induced nausea and vomiting: a multinational, randomized, double-blind, placebo-controlled trial in patients receiving high-dose cisplatin-the aprepitant protocol 052 study group. J Clin Oncol 2003;21:4112-4119.

9. Roeland EJ, Ruddy KJ, LeBlanc TW, et al. What the HEC? Clinician adherence to evidence-based antiemetic prophylaxis for highly emetogenic chemotherapy. J Natl Compr Canc Netw 2020;18:676-681.

10. Schwartzberg LS, Grunberg SM, Kris MG. Recent advances and updated guidelines in the management of chemotherapy-induced nausea and vomiting. Clin Adv Hematol Oncol 2011;9:1-14; quiz 15-6.

11. Clemons M, Bouganim N, Smith S, et al. Risk model-guided antiemetic prophylaxis vs physician's choice in patients receiving chemotherapy for early-stage breast cancer: a randomized clinical trial. JAMA Oncol 2016;2:225-231

12. Ng T, Mazzarello S, Wang Z, et al. Choice of study endpoint significantly impacts the results of breast cancer trials evaluating chemotherapy-induced nausea and vomiting. Breast Cancer Res Treat 2016;155:337-344.

13. $\mathrm{Ng} \mathrm{TL}$, Hutton $\mathrm{B}$, Clemons M. Chemotherapy-induced nausea and vomiting: time for more emphasis on nausea? Oncologist 2015;20: 576-583. 